\title{
Focal Muscle Vibration Changes the Architecture of the Medial Gastrocnemius Muscle in Persons With Limited Ankle Dorsiflexion
}

\author{
II-Young Moon ${ }^{1,2}$, PT, MSc, Jin-Seok Lim¹, PT, MSc, II-Woo Park ${ }^{1}$ PT, MSc, Chung-Hwi Yi ${ }^{3}$ PT, PhD \\ ${ }^{1}$ Department of Physical Therapy, The Graduate School, Yonsei University, ${ }^{2}$ Department of Rehabilitation Medicine, Wonju Severance \\ Christian Hospital, ${ }^{3}$ Department of Physical Therapy, College of Software and Digital Healthcare Convergence, Yonsei University, Wonju, \\ Korea
}

\author{
Article Info \\ Received December 31, 2021 \\ Revised January 18, 2022 \\ Accepted January 19, 2022 \\ Corresponding Author \\ Chung-Hwi Yi \\ E-mail: pteagle@yonsei.ac.kr \\ https://orcid.org/0000-0003-2554-8083
}

\section{Key Words}

Gastrocnemius

Muscle tonus

Ultrasonography

Vibration

\begin{abstract}
Background: The gastrocnemius tightness can easily occur. Gastrocnemius tightness results in gait disturbance. Thus, various interventions have been used to release a tight gastrocnemius muscle and improve gait performance. Moreover, focal muscle vibration (FMV) has recently been extensively researched in terms of tight muscle release and muscle performance. However, no study has investigated the effects of FMV application on medial gastrocnemius architectural changes.
\end{abstract}

Objects: In this study, we aimed to investigate the effects of FMV on medial gastrocnemius architecture in persons with limited ankle dorsiflexion.

Methods: Thirty one persons with $<10^{\circ}$ of passive ankle dorsiflexion participated in this study. We excluded persons with acute ankle injury within six months prior to study onset, a history of ankle fracture, leg length discrepancy greater than $2 \mathrm{~cm}$, no history of neurological dysfunction, or trauma affecting the lower limb. The specifications of the FMV motor were as follows: a fixed frequency (fast wave: $150 \mathrm{~Hz}$ ) and low amplitude $(0.3-0.5 \mathrm{~mm}$ peak to peak) of vibration; the motor was used to release the medial gastrocnemius for 15 minutes. Each participant completed three trials for 10 days; a 30-second rest period was provided between each trial. Medial gastrocnemius architectural parameters [muscle thickness (MT), fiber bundle length (FBL), and pennation angle (PA)] were measured via ultrasonography.

Results: MT significantly decreased after FMV application ( $p<0.05)$. FBL significantly increased from its baseline value after FMV application $(p<0.05)$. PA significantly decreased from its baseline value after FMV application ( $p<0.05)$.

Conclusion: FMV application may be advantageous in reducing medial gastrocnemius excitability following a decrease in the amount of contractile tissue. Furthermore, FMV application can be used as a stretching method to alter medial gastrocnemius architecture.

\section{INTRODUCTION}

The gastrocnemius is essential for walking and posture, and thus gastrocnemius tightness can easily occur [1]. A tight gastrocnemius results in gait disturbance and loss foot energy absorption [2]. Moreover, the medial gastrocnemius that contains more fibers per unit volume exerts a greater contribution to gait performance than the lateral gastrocnemius [3]. Various interventions, such as stretching [4], massage [5], and vibration [6] have been used to release gastrocnemius tightness and improve gait performance [7]. Moreover, recently, the effect of focal muscle vibration (FMV) application on gastrocnemius tightness release, and muscle performance has been extensively investigated [8].

FMV, a non-invasive technique through which targeted vibration is easily applied to specific muscles, facilitates the proprioceptive nervous system [9]. A vibration motor and actuator drive were used to operate the FMV device. The vibration motor, connecting the eccentric load to the rotor of the motor, generates an unbalanced rotation force [10]. This rotation force can be easily controlled using a fixed frequency and amplitude of the FMV device. Due to these advantages, many therapists use the FMV device to prolong the exposure of specific muscles to vibrations to reduce muscle tightness [11,12]. 
Ultrasonography is a useful tool for assessing muscle architectural properties, such as muscle thickness (MT), fiber bundle length (FBL), and pennation angle (PA). Muscle architecture can be used to determinate muscle function and force-generating capacity, which forms the basis for physiological movement [13]. Thus, the use of ultrasonography to measure muscle architectural changes is an important factor for enhancing human performance [14].

Few studies have compared the effects of FMV in exercise performance; further, other studies have examined the changes in muscle properties based on the joint angle $[15,16]$. However, no study has investigated the effects of FMV application on medial gastrocnemius architectural changes. Therefore, in this study, we aimed to assess, using ultrasonography, the medial gastrocnemius architecture via FMV application in participants with limited ankle dorsiflexion.

\section{MATERIALS AND METHODS}

\section{Participants}

A priori power analysis using G-power software (ver. 3.1.9.4; Franz Faul, Kiel University, Kiel, Germany) was used to estimate the sample size (Wilcoxon signed-rank test; effect size $=0.5$, alpha level $=0.05$, power $=0.80$ ), the estimated sample size was 31 . We included active participants who volunteered to participate in this study and had a passive ankle dorsiflexion range of motion $(\mathrm{ROM})<10^{\circ}$. Novacheck [17] demonstrated that a passive ankle dorsiflexion ROM of at least $10^{\circ}$ is required for sufficient ambulation. Moreover, we excluded patients who presented with acute ankle injury within six months prior to the study onset, a history of ankle fracture, leg length

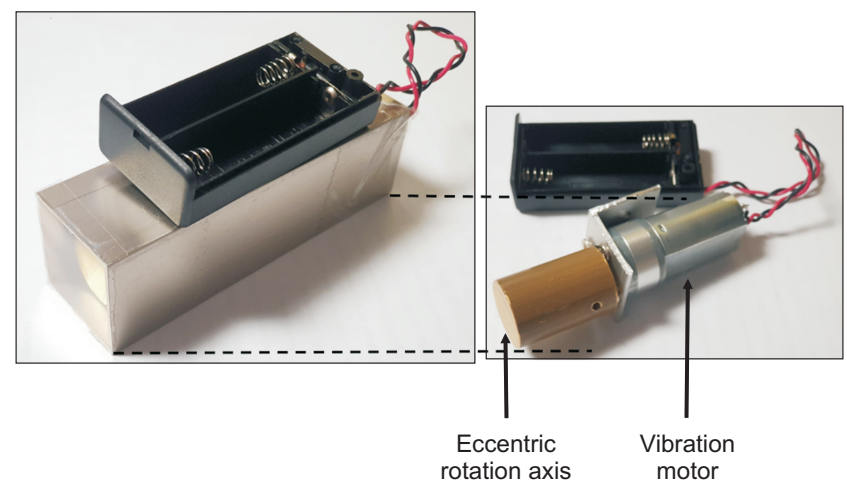

Figure 1. Specification of the focal muscle vibration motor with a fixed high frequency (fast wave: $150 \mathrm{~Hz}$ ) and low amplitude $(0.3-0.5 \mathrm{~mm}$ peak to peak). discrepancy greater than $2 \mathrm{~cm}$ [18], no history of neurological dysfunction, or trauma affecting the lower limb [19]. All volunteered participants provided written informed consent, and the study was approved by Institutional Review Board of the Graduate School, Yonsei University, Wonju (IRB no. 1041849202103-BM-042-01).

\section{Focal Muscle Vibration Therapy}

In this study, a custom-made FMV device (UVTEC Inc., Incheon, Korea), using an eccentric rotor vibration motor, was used to release medial gastrocnemius tightness for 15 minutes (the ratio between right and left medial gastrocnemius was 29 to 2). Each participant completed three trials for 10 days, and a 30-second rest period was provided between each trial. The medial gastrocnemius architectural parameters were measured via ultrasonography. The specifications of the vibration motor for FMV application were as follows: a fixed frequency (fast wave: $150 \mathrm{~Hz}$ ) and low-amplitude (0.3-0.5 mm peak to peak) (Figure 1). The FMV device was placed on the medial gastrocnemius, around the upper third of the posterior lower leg (Figure 2). The directions of the anchor and strap were perpendicular to the shaft of the motor, and the vector of the vibratory stimulus was parallel to the muscle fiber.

\section{Outcome Measures}

\section{1) Medial gastrocnemius architecture}

An ultrasound scanner with a 7.5-MHz linear transducer (SonoAce x8, Samsung Medison Co., Ltd., Seoul, Korea) was used

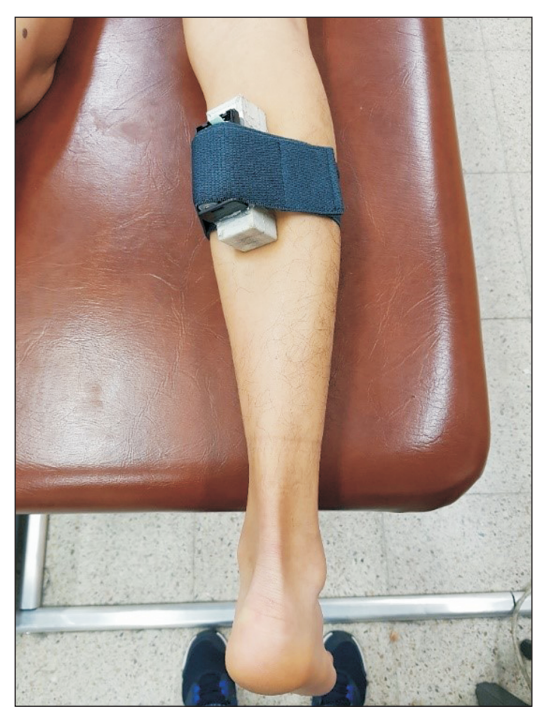

Figure 2. Focal muscle vibrator device placement on the medial gastrocnemius. 
to measure the medial gastrocnemius architectural parameters. Using the protocol provided by a previous study, medial gastrocnemius architectural images were obtained [20]. To ensure the consistency of the measurements, all images were obtained by a physical therapist, who was trained to administer the medial gastrocnemius ultrasound imaging protocol. The ultrasound probe was placed on a quarter proximal to the distance between the popliteal crease and the center of the lateral malleolus (Figure 3). Image acquisitions from medial gastrocnemius architecture were used in the mean value of three trials. The intra-reliability of the ultrasound used in our study was calculated for comparison with previous studies [20,21].

MT was measured as the longest distance between the superficial and deep fascias of the medial gastrocnemius. The PA was defined as the angle made by the insertion of the muscle fiber fascicles into deeper aponeurosis (Figure 4). The FBL was defined as the length of the fascicular path between the superficial and deep muscular fascias and was estimated using the following mathematical formula [21].

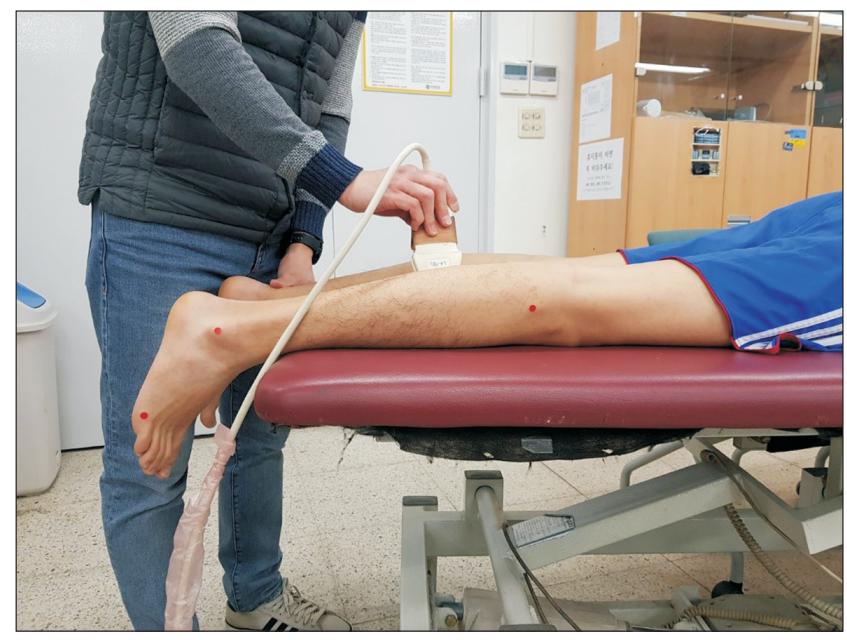

Figure 3. Ultrasound probe positioning for the medial gastrocnemius.

$$
\mathrm{FBL}=\frac{\mathrm{MT}}{\operatorname{Sin}(\mathrm{PA})}
$$

\section{Statistical Analysis}

Statistical analysis was performed using IBM SPSS Statistics for Windows version 24.0 (IBM Co., Armonk, NY, USA) and the level of statistical significance $(\alpha=0.05)$. The Wilcoxon signedrank test was performed to compare the dependent variables (MT, PA, and FBL) before and after FMV application.

\section{RESULTS}

\section{Participants' Demographics}

Thirty one healthy university participants (age $=23.9 \pm 3.74$ years; height $=171.6 \pm 7.19 \mathrm{~cm}$; body mass index $=70.9 \pm$ $14.23 \mathrm{~kg} / \mathrm{m}^{2}$ ) were included in this study.

\section{Muscle Architecture}

The medial gastrocnemius architectural changes evaluated using ultrasonography are shown in Figure 5. MT significantly

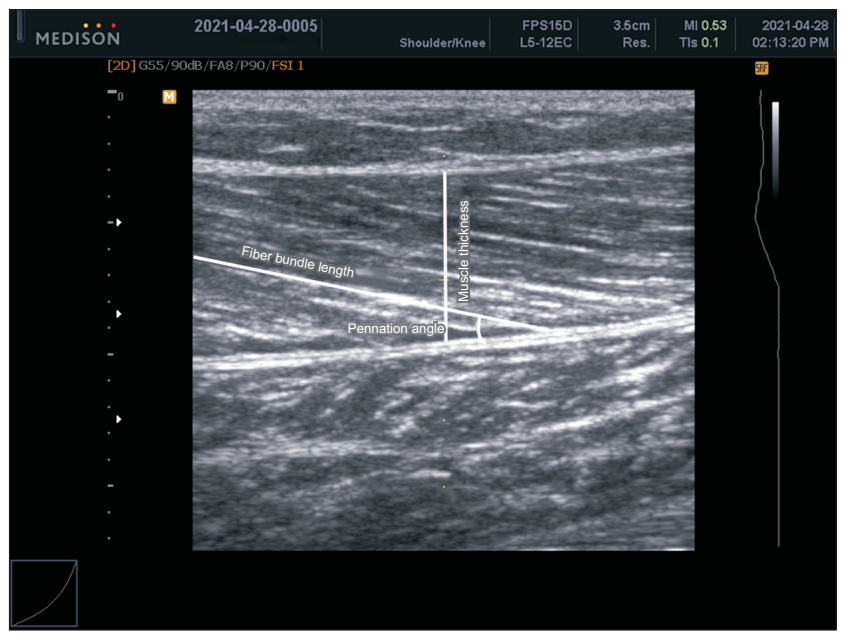

Figure 4. Ultrasound image of the medial gastrocnemius.
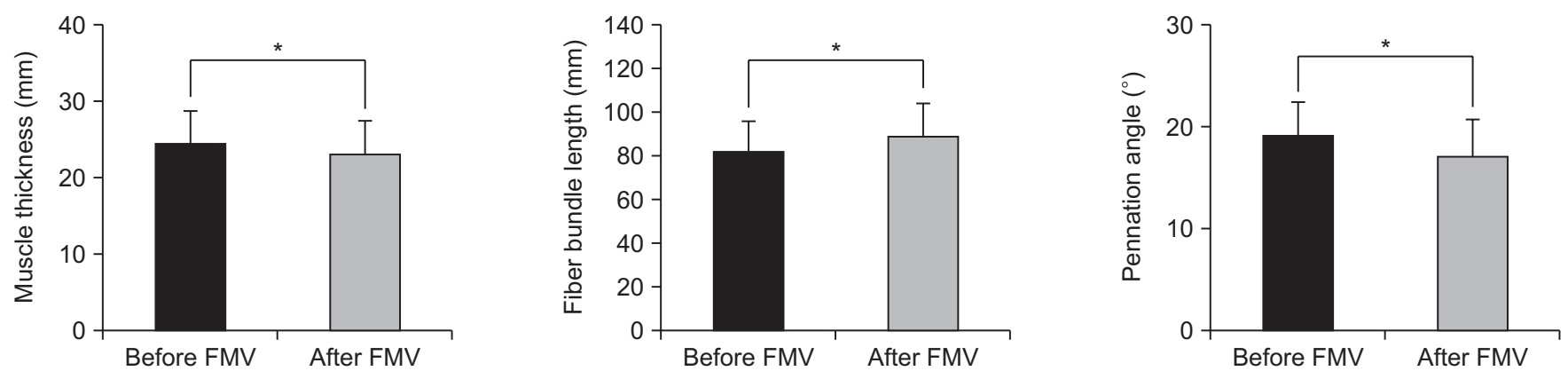

Figure 5. Ultrasonographic data for medial gastrocnemius architecture. Error bars indicate standard deviation. FMV, focal muscle vibration. ${ }^{*} p<0.05$. 
Table 1. Wilcoxon signed-rank test between before and after FMV application

\begin{tabular}{lll}
\hline \multicolumn{1}{c}{ Variable } & \multicolumn{1}{c}{ Before FMV } & \multicolumn{1}{c}{ After FMV } \\
\hline Muscle thickness $(\mathrm{mm})$ & $24.38 \pm 4.50$ & $22.90 \pm 4.63^{*}$ \\
Fiber bundle length $(\mathrm{mm})$ & $81.73 \pm 14.45$ & $88.66 \pm 15.76^{*}$ \\
Pennation angle $\left(^{\circ}\right)$ & $19.12 \pm 3.35$ & $17.02 \pm 3.76^{*}$ \\
\hline
\end{tabular}

Values are presented as mean \pm standard deviation. FMV, focal muscle vibration. ${ }^{*} p<0.05$.

decreased from $24.4 \pm 4.49$ to $22.9 \pm 4.63 \mathrm{~mm}(\mathrm{p}<0.05)$ after FMV application. The FBL significantly increased from 81.7 \pm 14.45 to $88.7 \pm 15.76 \mathrm{~mm}(\mathrm{p}<0.05)$ after FMV application. The PA significantly decreased after FMV application from 19.1 $\pm 3.35^{\circ}$ to $17.0 \pm 3.76^{\circ}(\mathrm{p}<0.05)$ compared with the baseline value (Table 1).

\section{DISCUSSION}

This is the first study to quantify changes in muscle architectural parameters (MT, FBL, and PA) using an FMV device in participants with limited ankle dorsiflexion. Significant differences were found in MT, FBL, and PA after applying FMV. These findings indicate that FMV application may be advantageous in altering the amount of contractile tissue in the medial gastrocnemius.

In this study, MT significantly decreased after FMV application. This implies that FMV application allowed for medial gastrocnemius release by decreasing MT compared with the initial position. MT is commonly used for predicting the maximum force-generating capacity, which is defined by muscle activity; moreover, MT has been significantly correlated with the anatomical cross-sectional area [22]. Hebert et al. [23] reported a positive linear relationship between MT and abdominal electromyographic (EMG) activity during ultrasound imaging. Hides et al. [24] showed good to high correlation between MT and abdominal EMG activity in a magnetic resonance imaging study. Thus, MT is often used as an index of muscle activity. A fixed high-frequency (fast wave: $150 \mathrm{~Hz}$ ) and low-amplitude (0.3-0.5 mm peak to peak) FMV was used to reduce the muscle excitability and to restrict the stimuli to specific muscles [25]. The stimuli provide the proprioceptive input by activating the neural pathway from the muscle spindle [8]. This proprioceptive input adjusts the intra-cortical inhibitory and facilitatory networks, thereby altering the excitability of the corticospinal pathway. Therefore, a localized application of FMV to the me- dial gastrocnemius reduces the focal muscle excitability.

The FBL is the most important muscle architectural parameter and indicates the number of sarcomeres in a series [26]. Thus, a decrease in muscle contraction velocity or range of muscle fiber excursion influences the FBL [27]. An increase in FBL indicates a decrease in the amount of contractile tissue. Ribot-Ciscar et al. [28] reported that FMV application on specific muscles creates centrally localized neural changes that may decrease the spontaneous firing rate in the muscle spindle of primary sensory nerve endings, thereby changing the length-tension property of the muscle. In this study, FBL significantly increased after FMV application. This result is clinically useful, as it shows that FMV application is effective in releasing the medial gastrocnemius, and thus may effectively influence gastrocnemius stiffness.

The larger the PA, the greater the muscle contractility, and this increases the physiological cross-sectional area of the muscle [29]. Thus, an increase in PA indicates an increase in the muscle's capacity to produce force. In our study, the PA significantly decreased from the baseline value after FMV application. Therefore, applying FMV on the medial gastrocnemius may decrease the muscle's capacity for force productivity. Pilch et al. [30] reported that vibration application on a muscle plays a significant role in increasing tissue temperature. This tissue temperature alteration may be associated with improved blood flow and reduced muscle viscosity [31]. Malanga et al. [32] demonstrated that an increased local blood circulation reduces muscle spasm or muscle activity. Considering a heating mechanism, the local application of heat to a muscle is commonly recommended to strengthen the efficacy of muscle stretching [33]. Thus, FMV application can be used as a means to enhance the efficacy of muscle stretching using a neural pathway mechanism and heating effect.

This study has a few limitations. First, the study participants were generally vicenarians. This limited age group impedes the generalizability of the results. In further studies, a variety of age groups would be required to evaluate the muscle architectural properties. Second, we only investigated muscle architecture; functional or motion analysis (such as ROM, balance, or walking) was not performed. Further research will focus on the abovementioned variables. 


\section{CONCLUSIONS}

In the present study, we investigated muscle architectural properties (MT, FBL, and PA) after FMV application in participants with limited ankle dorsiflexion. The findings suggest that FMV may have an advantage in reducing the medial gastrocnemius excitability following a decrease in the amount of contractile tissue. Furthermore, FMV application can be used as a stretching method for altering medial gastrocnemius architecture.

\section{ACKNOWLEDGEMENTS}

This study was supported by the "Brain Korea 21 FOUR Project", the Korean Research Foundation for Department of Physical Therapy in the Graduate School of Yonsei University.

\section{CONFLICTS OF INTEREST}

No potential conflict of interest relevant to this article was reported.

\section{AUTHOR CONTRIBUTIONS}

Conceptualization: IYM, CHY. Data curation: IYM, JSL, IWP.

Formal analysis: JSL, IWP. Funding acquisition: JSL, CHY. Investigation: IYM, JSL, IWP, CHY. Methodology: IYM, JSL, IWP, CHY. Project administration: CHY. Resources: IYM, IWP. Software: IYM. Supervision: IWP, CHY. Validation: IYM, JSL. Visualization: IWP, CHY. Writing - original draft: IYM, JSL. Writing - review \& editing: IWP, CHY.

\section{ORCID}

Il-Young Moon, https://orcid.org/0000-0001-6457-8125

Jin-Seok Lim, https://orcid.org/0000-0002-9965-9619

Il-Woo Park, https://orcid.org/0000-0002-3821-8916

\section{REFERENCES}

1. DiGiovanni CW, Kuo R, Tejwani N, Price R, Hansen ST Jr, Cziernecki J, et al. Isolated gastrocnemius tightness. J Bone Joint Surg Am 2002;84(6):962-70.

2. Rao SR, Saltzman CL, Wilken J, Yak HJ. Increased passive ankle stiffness and reduced dorsiflexion range of motion in individuals with diabetes mellitus. Foot Ankle Int 2006;27(8):61722.

3. Kawakami Y, Ichinose Y, Fukunaga T. Architectural and functional features of human triceps surae muscles during contraction. J Appl Physiol (1985) 1998;85(2):398-404.

4. Andersen JC. Stretching before and after exercise: effect on muscle soreness and injury risk. J Athl Train 2005;40(3):21820.

5. Ajimsha MS, Al-Mudahka NR, Al-Madzhar JA. Effectiveness of myofascial release: systematic review of randomized controlled trials. J Bodyw Mov Ther 2015;19(1):102-12.

6. Calabrò RS, Naro A, Russo M, Milardi D, Leo A, Filoni S, et al. Is two better than one? Muscle vibration plus robotic rehabilitation to improve upper limb spasticity and function: a pilot randomized controlled trial. PLoS One 2017;12(10):e0185936.

7. Lavery LA, Armstrong DG, Boulton AJ; Diabetex Research Group. Ankle equinus deformity and its relationship to high plantar pressure in a large population with diabetes mellitus. J Am Podiatr Med Assoc 2002;92(9):479-82.

8. Paoloni M, Tavernese E, Fini M, Sale P, Franceschini M, Santilli $\mathrm{V}$, et al. Segmental muscle vibration modifies muscle activation during reaching in chronic stroke: a pilot study. NeuroRehabilitation 2014;35(3):405-14.

9. Celletti C, Camerota F. Preliminary evidence of focal muscle vibration effects on spasticity due to cerebral palsy in a small sample of Italian children. Clin Ter 2011;162(5):e125-8.

10. Celik O, O'Malley MK, Brent Gillespie R, Shewokis PA, Contreras-Vidal JL. Compact and low-cost tendon vibrator for inducing proprioceptive illusions. Paper presented at: World Haptics 2009 - 3rd Joint EuroHaptics Conference and Symposium on Haptic Interfaces for Virtual Environment and Teleoperator Systems; 2009 Mar 18-20; Salt Lake City, UT, USA. Danvers (MA): IEEE, 2009. p. 623-4.

11. Avanzino L, Pelosin E, Abbruzzese G, Bassolino M, Pozzo T, Bove M. Shaping motor cortex plasticity through proprioception. Cereb Cortex 2014;24(10):2807-14.

12. McNickle E, Carson RG. Paired associative transcranial alternating current stimulation increases the excitability of corticospinal projections in humans. J Physiol 2015;593(7):164966.

13. Fukutani A, Kurihara T. Comparison of the muscle fascicle length between resistance-trained and untrained individuals: cross-sectional observation. Springerplus 2015;4:341. 
14. de Boer MD, Seynnes OR, di Prampero PE, Pisot R, Mekjavić IB, Biolo G, et al. Effect of 5 weeks horizontal bed rest on human muscle thickness and architecture of weight bearing and nonweight bearing muscles. Eur J Appl Physiol 2008;104(2):4017.

15. Bakhtiary AH, Fatemi E, Khalili MA, Ghorbani R. Localised application of vibration improves passive knee extension in women with apparent reduced hamstring extensibility: a randomised trial. J Physiother 2011;57(3):165-71.

16. Fagnani F, Giombini A, Di Cesare A, Pigozzi F, Di Salvo V. The effects of a whole-body vibration program on muscle performance and flexibility in female athletes. Am J Phys Med Rehabil 2006;85(12):956-62.

17. Novacheck TF. The biomechanics of running. Gait Posture 1998;7(1):77-95.

18. Dinh NV, Freeman H, Granger J, Wong S, Johanson M. Calf stretching in non-weight bearing versus weight bearing. Int J Sports Med 2011;32(3):205-10

19. Johanson MA, DeArment A, Hines K, Riley E, Martin M, Thomas J, et al. The effect of subtalar joint position on dorsiflexion of the ankle/rearfoot versus midfoot/forefoot during gastrocnemius stretching. Foot Ankle Int 2014;35(1):63-70.

20. Samukawa M, Hattori M, Sugama N, Takeda N. The effects of dynamic stretching on plantar flexor muscle-tendon tissue properties. Man Ther 2011;16(6):618-22.

21. Kumagai K, Abe T, Brechue WF, Ryushi T, Takano S, Mizuno M. Sprint performance is related to muscle fascicle length in male 100-m sprinters. J Appl Physiol (1985) 2000;88(3):8116.

22. Moreau NG, Simpson KN, Teefey SA, Damiano DL. Muscle architecture predicts maximum strength and is related to activity levels in cerebral palsy. Phys Ther 2010;90(11):1619-30.

23. Hebert JJ, Koppenhaver SL, Parent EC, Fritz JM. A systematic review of the reliability of rehabilitative ultrasound imaging for the quantitative assessment of the abdominal and lumbar trunk muscles. Spine (Phila Pa 1976) 2009;34(23):E848-56.
24. Hides JA, Belavý DL, Cassar L, Williams M, Wilson SJ, Richardson CA. Altered response of the anterolateral abdominal muscles to simulated weight-bearing in subjects with low back pain. Eur Spine J 2009;18(3):410-8.

25. Murillo N, Valls-Sole J, Vidal J, Opisso E, Medina J, Kumru H. Focal vibration in neurorehabilitation. Eur J Phys Rehabil Med 2014;50(2):231-42.

26. Park JE, Seong YJ, Kim ES, Park D, Lee Y, Park H, et al. Architectural changes in the medial gastrocnemius on sonography after nerve ablation in healthy adults. Yonsei Med J 2019;60(9):876-81.

27. Lieber RL. Skeletal muscle architecture: implications for muscle function and surgical tendon transfer. J Hand Ther 1993;6(2):105-13.

28. Ribot-Ciscar E, Rossi-Durand C, Roll JP. Muscle spindle activity following muscle tendon vibration in man. Neurosci Lett 1998;258(3):147-50.

29. Moreau NG, Teefey SA, Damiano DL. In vivo muscle architecture and size of the rectus femoris and vastus lateralis in children and adolescents with cerebral palsy. Dev Med Child Neurol 2009;51(10):800-6.

30. Pilch W, Czerwińska-Ledwig 0, Chitryniewicz-Rostek J, Nastałek M, Krężałek P, Jędrychowska D, et al. The impact of vibration therapy interventions on skin condition and skin temperature changes in young women with lipodystrophy: a pilot study. Evid Based Complement Alternat Med 2019;2019:8436325.

31. Taylor DC, Dalton JD Jr, Seaber AV, Garrett WE Jr. Viscoelastic properties of muscle-tendon units. The biomechanical effects of stretching. Am J Sports Med 1990;18(3):300-9.

32. Malanga GA, Yan N, Stark J. Mechanisms and efficacy of heat and cold therapies for musculoskeletal injury. Postgrad Med 2015;127(1):57-65.

33. Nakano J, Yamabayashi C, Scott A, Reid WD. The effect of heat applied with stretch to increase range of motion: a systematic review. Phys Ther Sport 2012;13(3):180-8. 